\title{
Propriedades psicométricas de instrumento para avaliação de egressos de cursos de graduação em enfermagem
}

Psychometric properties of an assessment instrument of graduates of nursing undergraduate courses Propiedades psicométricas de un instrumento de evaluación de egresados de carreras de enfermería

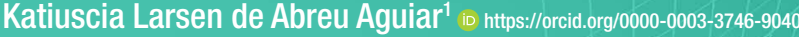 \\ Maria Aparecida Vieira² ${ }^{2}$ it htps://orcid.org/0000-0002-7921-4049 \\ Edvane Birelo Lopes De Domenico ${ }^{3}$ ib https://orcid.org/0000-0001-7455-1727
}

Como citar: Aguiar KL, Vieira MA, De Domenico EB. Propriedades psicométricas de instrumento para avaliação de egressos de cursos de graduação em enfermagem. Acta Paul Enferm. 2021;34:eAPE001825.

DOI http://dx.doi.org/10.37689/actaape/2021A0001825

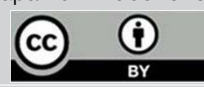

Descritores

Educação em enfermagem; Estudos de validação; Estudos de avaliação; Avaliação em enfermagem

Keywords

Nursing education; Validation studies; Evaluation studies; Nursing evaluation

Descriptores

Educación en enfermería; Estudios de validación Estudios de evaluación; Evaluación de enfermería

\section{Submetido}

14 de Julho de 2020

Aceito

1 de Março de 2021

Autor correspondente

Katiuscia Larsen de Abreu Aguiar Rodovia BR 364, Km 04 - 69920-900,

Distrito Industrial, Rio Branco, AC, Brasil.

E-mail: katiuscia.larsen@gmail.com

\section{Resumo}

Objetivo: Validar psicometricamente o Instrumento Avaliação de Egressos de Enfermagem.

Métodos: Estudo transversal, quantitativo, realizado em três Cursos de Graduação em Enfermagem localizados nos Estados de São Paulo, Minas Gerais e Acre, Brasil, com 446 participantes. A confiabilidade foi testada pelo alfa de Cronbach (consistência interna e reprodutibilidade) e a validade de construto foi obtida pela análise fatorial exploratória e pela análise dos componentes principais.

Resultados: 0 valor do coeficiente Alfa de Cronbach total do instrumento foi de 0,98; os coeficientes de correlação intraclasse total de (94\%), evidenciando forte concordância entre as medições. Na análise fatorial, KMO foi de 0,971 e 0 teste de esfericidade de Bartlett apresentou resultados significativos $(p<0,001)$ para todos os construtos e as matrizes de correlação entre os itens de cada construto.

Conclusão: As análises psicométricas mostraram resultados favoráveis, evidenciando forte consistência interna do instrumento. 0 Instrumento Avaliação de Egressos de Enfermagem é válido e confiável para a avaliação da formação profissional do egresso bacharel em Enfermagem.

\section{Abstract}

Objective: Psychometric validation of the Assessment Instrument of Nursing Graduates.

Methods: Cross-sectional, quantitative study conducted in three Nursing Undergraduate Courses located in the states of São Paulo, Minas Gerais and Acre, Brazil, with 446 participants. Reliability was tested by Cronbach's alpha (internal consistency and reproducibility) and construct validity was obtained by exploratory factor analysis and principal component analysis.

Results: The overall Cronbach's alpha coefficient of the instrument was 0.98 ; the overall intraclass correlation coefficient (94\%) showed strong agreement between measurements. In factor analysis, KM0 was 0.971 and the Bartlett's sphericity test showed significant results $(p<0.001)$ for all constructs and correlation matrices between the items of each construct.

Conclusion: The psychometric analyzes showed favorable results and a strong internal consistency of the instrument. The Assessment Instrument of Nursing Graduates is valid and reliable to evaluate the professional education of Nursing graduates.

\section{Resumen}

Objetivo: Validar psicométricamente el instrumento Evaluación de Egresados de Enfermería.

'Centro de Ciências da Saúde e do Desporto, Universidade Federal do Acre, Rio Branco, AC, Brasil.

2Departamento de Enfermagem, Universidade Estadual de Montes Claros, Montes Claros, MG, Brasil.

${ }^{3}$ Departamento de Enfermagem Clínica e Cirúrgica, Escola Paulista de Enfermagem, Universidade Federal de São Paulo, São Paulo, SP, Brasil.

Conflitos de interesse: Esse manuscrito foi extraído da tese "Validação Externa do Instrumento de valiação de Egressos de Enfermagem IAE-ENF: Estudo multicêntrico, 2019", que foi apresentado à Universidade Federal de São Paulo/UNIFESP. Embora Domenico EBL seja Editora Associada da Acta Paulista de Enfermagem, informamos que ela não teve acesso ao processo de avaliação do referido manuscrito, tampouco dos avaliadores que revisaram 0 artigo. 
Métodos: Estudio transversal, cuantitativo, realizado en tres carreras de grado de enfermería ubicadas en los estados de São Paulo, Minas Gerais y Acre, Brasil, con 446 participantes. La fiabilidad fue probada mediante el alfa de Cronbach (consistencia interna y reproducibilidad) y la validez del constructo se obtuvo con el análisis factorial exploratorio y el análisis de los componentes principales.

Resultados: El valor del coeficiente alfa de Cronbach total del instrumento fue de 0,98. Los coeficientes de correlación intraclase totales fueron del 94 \%, lo que evidencia fuerte concordancia entre las mediciones. En el análisis factorial, el KMO fue de 0,971 y la prueba de esfericidad de Bartlett presentó resultados significativos $(p<0,001)$ en todos los constructos y las matrices de correlación entre los ítems de cada constructo.

Conclusión: Los análisis psicométricos mostraron resultados favorables, lo que evidencia una fuerte consistencia interna del instrumento. El instrumento Evaluación de Egresados de Enfermería es válido y confiable para evaluar la formación profesional del egresado Licenciado en Enfermería.

\section{Introdução}

A avaliação institucional universitária é uma valiosa oportunidade de voltar o olhar e a crítica sobre si, reconhecendo a importância que a instituição de ensino superior (IES) tem como polo de construção e disseminação de conhecimento. Quando realizada de forma criteriosa e quanto se torna uma prática cultural, é uma ferramenta diagnóstica preciosa para a qualidade, geradora de dados a serem utilizados no aprimoramento ou na reorientação do processo educacional, seja qual for o cenário aplicado, aula, disciplina, curso, instituição de ensino. ${ }^{(1)}$

A Educação Superior tem o objetivo de diplomar, em diferentes áreas de conhecimento, sujeitos que estejam aptos a serem inseridos em setores profissionais para o desenvolvimento da sociedade brasileira, incentivando o permanente desejo de aperfeiçoamento cultural e profissional. Em âmbito nacional, a avaliação do Ensino Superior está sob o controle do Instituto Nacional de Pesquisas Educacionais (INEP) do Ministério da Educação (MEC) que regulamenta, fiscaliza e reforça a avaliação contínua e sistemática das IES. (2)

Na perspectiva da formação profissional universitária e, especificamente, sobre a da Enfermagem, área profissional com grande contingente de formandos em saúde, é necessário avaliar o processo educacional nos anos de formação, bem como o produto da mesma, buscando-se, no egresso, averiguar se o papel social da IES está sendo cumprido junto à sociedade e se o profissional recém-formado está capacitado para exercer o que lhe foi outorgado. ${ }^{(3)}$

Nesse contexto, a avaliação do egresso deve verificar se as atividades acadêmicas elaboradas coletivamente pelos gestores institucionais estão sendo efetivamente acolhidas com eficiência e com qualidade, considerando os ideais da profissão, da so- ciedade e do mercado de trabalho. ${ }^{(4,5)}$ Embora seja complexa a avaliação do egresso, a intencionalidade fundamental, na prática, é conhecer como as IES têm seguido os princípios básicos que direcionam a Educação Superior, incluindo o da Enfermagem.

Em estudos anteriores propóem-se instrumentos desenvolvidos com a finalidade de "conhecer" o egresso quanto à sua formação profissional e empregabilidade, com vistas à obtenção de informaçóes para embasar mais concretamente as decisóes pedagógicas e administrativas. ${ }^{(6-9)}$ No entanto, observa-se a escassez de estudos com o objetivo de averiguar a construção de conhecimentos requeridos para o exercício das competências e habilidades gerais exigidas pelas Diretrizes Curriculares Nacionais para a área da Enfermagem (DCN/ENF). ${ }^{(10)}$

Assim, a consagração de um modelo complementar de avaliação do processo educacional que ateste quantitativamente e qualitativamente o ensino ofertado faz-se tão importante quanto expandir a Educação Superior em território nacional. A padronização de instrumentos válidos e confiáveis é de fundamental importância para certificar a qualidade da educação superior como um todo. ${ }^{(4,11)}$

Nesse sentido, foi criado instrumento com a finalidade de avaliar egressos de Cursos de Graduação em Enfermagem a partir das DCN/ENF intitulado Instrumento para a Avaliação de Egressos de Cursos de Graduação em Enfermagem (IAE-ENF), cujo processo de validação de conteúdo foi cumprido e a confiabilidade testada pelo alfa de Cronbach $(0,750)$ sem que houvesse, entretanto, a validação externa. ${ }^{(12)}$

Considerando a importância da avaliação de egressos, a atual pesquisa justifica-se pela necessidade de complementar o estudo anterior, aprofundando a avaliação das propriedades psicométricas do IAEENF em uma população multicêntrica de egressos de Cursos de Graduação em Enfermagem, para que 
este se torne um instrumento válido e confiável, permitindo assim, a verificação da convergência entre as atividades acadêmicas, os pressupostos contidos nas DCN/ENF e o produto da formação. ${ }^{(12)}$ Objetivou-se portanto, validar psicometricamente o Instrumento Avaliação de Egressos de Enfermagem.

\section{Métodos}

Pesquisa do tipo metodológico, transversal, de abordagem quantitativa e multicêntrica.

\section{Aspectos éticos}

$\mathrm{O}$ estudo atendeu às normas que envolvem aspectos éticos em pesquisas envolvendo seres humanos, aprovado pelo Comitê de Ética em Pesquisas sob o Parecer no 0977/2016 e Certificado de Apresentação e Apreciação Ética (CAAE), n ${ }^{\circ}$ 57869516.7.0000.5505.

\section{Local e população de estudo}

Os locais de estudo incluíram três IES brasileiras: Universidade Federal do Acre (IES-1), situada na regiáo norte do Brasil; Universidade Estadual de Montes Claros (IES-2) e a Universidade Federal de São Paulo (IES-3), ambas na regiáo sudeste. A escolha pelo estudo multicêntrico deu-se pela possibilidade de comparar dados provenientes de diferentes contextos educacionais, sociais, econômicos e culturais. A seleção das três universidades deu-se por conveniência, uma vez que são locais de trabalho das pesquisadoras. As instituiçóes participantes consentiram na realização da pesquisa, bem como na divulgação nominal das mesmas, por meio da assinatura de uma Carta de Anuência.

Para o cálculo do tamanho da amostra (n), estimando-se o nível de confiança de $95 \%$ e nível de significância de 0,05 , levou-se em consideração o número total de egressos titulados de 2013 a 2017 (n: 603), disponibilizados pelas três secretarias acadêmicas dos cursos participantes. O tamanho da amostra foi calculado usando o sistema operacional OpenEpi ${ }^{\oplus}$ versão $2{ }^{(13)}$ De acordo com literatura especializada, para cada pergunta do questionário é preciso haver, no mínimo, cinco sujeitos. ${ }^{(14)}$ Como o IAE-ENF contém 87 questóes, o número amostral estipulado foi de 435 egressos, mas um número superior, de 446 egressos participaram, sendo 75 egressos da IES-1, 341 egressos da IES-3/SP e 187 egressos da IES-2.

Os critérios de inclusão adotados na pesquisa foram: ser egresso do Curso de Graduação em Enfermagem de uma das três instituições participantes da pesquisa; ter concluído sua formação no período de 2013 a 2017. Critérios de exclusão: não responder e devolver todos os instrumentos solicitados, devidamente preenchidos, nos prazos estipulados.

\section{Instrumento e procedimentos de coleta de dados} Os 87 itens do IAE-ENF IAE-Enf estão divididos em três dimensões: Dimensão 1- Caracterização do Egresso: corresponde aos dados sociodemográficos como: nacionalidade, etnia, sexo, ano de nascimento, ano de conclusão do curso, natureza jurídica da Instituição, turno, atividades extracurriculares e titulação máxima; Dimensão 2- Avaliação da Formação Profissional: com 8 questóes fechadas baseadas nas DCN/ENF para a avaliação do processo de formação profissional e conhecimentos específicos da enfermagem; Dimensão 3- Avaliação da Atuação Profissional (na interface com a graduação): 6(seis) questôes fechadas sobre o tipo de formação recebida, satisfação quanto à remuneração e à profissão de enfermeiro, e 2 (duas) questóes abertas sobre os pontos positivos e negativos do curso de enfermagem que realizou.

A coleta de dados ocorreu entre junho de 2017, buscando-se os egressos de 2013 a 2016, e finalizou em junho de 2018, quando obteve-se os dados dos formandos de 2017. Os dados foram coletados por meio de um questionário eletrônico estruturado disponível em uma página na internet, utilizando-se uma versão avançada do software SurveyMonkey .

A captação dos respondentes ocorreu através de ampla divulgação por meio de correio eletrônico e redes sociais e por fim pela técnica bola de neve (snowball sampling). Esta técnica, não foi previamente determinada, mas foi necessária ao longo do processo de coleta de dados. Consiste na captaçáo de respondentes pertencentes à mesma população-alvo do estudo em que uma pessoa do grupo indica outros participantes referentes ao mesmo grupo e assim sucessivamente. ${ }^{(15)}$ 


\section{Análise dos resultados}

Após a conclusão da fase de coleta de dados, os dados do instrumento foram armazenados no item "Análise de Resultados" do software on-line SurveyMonkey ${ }^{\circ} \mathrm{e}$ foram tabulados em uma planilha de Microsoft Excel.

A avaliação das propriedades psicométricas do IAE-ENF seguiu passos estabelecidos em literatura, que consistem em testes de confiabilidade e validade para comprovar a autenticidade de um instrumento. ${ }^{(16)}$ Cabe esclarecer que o estudo de confiabilidade foi realizado nas três dimensóes e a validade restringiu-se às dimensóes 2 e 3 por ensejarem os preceitos contidos nas DCN/ENF.

A confiabilidade do IAE-ENF foi analisada por meio da reprodutibilidade por meio do teste-reteste com um intervalo de 7 a 15 dias entre as aplicaçóes e a amostra foi composta de 58 egressos. Para testar a confiabilidade interna do IAE-ENF, foi utilizado o Alfa de Cronbach, no qual o valor mínimo aceitável para o alfa foi de 0,70 e o valor máximo esperado de $0,90 .^{(16,17)}$

A validaçáo de construto foi realizada por meio da análise fatorial exploratória. Para isso, o primeiro passo foi verificar a adequabilidade do conjunto de itens contidos no IAE-ENF por meio da realização dos testes de KMO (Kaiser-Meyer-Olkin) e do teste de Bartlett (BTS). Os valores esperados para os testes de KMO são entre 0,5 e 1 e de BTS, p menor que 0,5 . $^{(18,19)}$

Em seguida, foi realizada a análise dos componentes principais, sendo selecionados os fatores que obtiveram valores próprios (eingenvalues) ou autovalores (variância total explicada para cada fator) maiores que um. A extração dos fatores principais foi executada depois da rotaçáo ortogonal Varimax e critério de Kaiser. ${ }^{(18)}$

\section{Resultados}

A confiabilidade do IAE-ENF foi testada pelo coeficiente Alfa de Cronbach nas três dimensóes e o valor total encontrado foi de 0,98 , o que caracteriza uma alta confiabilidade. Nas dimensóes 2 e 3, os valores encontrados pelo mesmo coeficiente foram maiores do que 70\%. Especificamente, foi observado um alfa de Cronbach de 0,98\% para a dimensão avaliação da formaçáo e de $78 \%$ para a dimensão atuaçáo profissional. Por meio desses resultados, foi possível afirmar que os itens são homogêneos e que o instrumento IAE-ENF mede consistentemente a característica para a qual foi proposto (Figura 1).

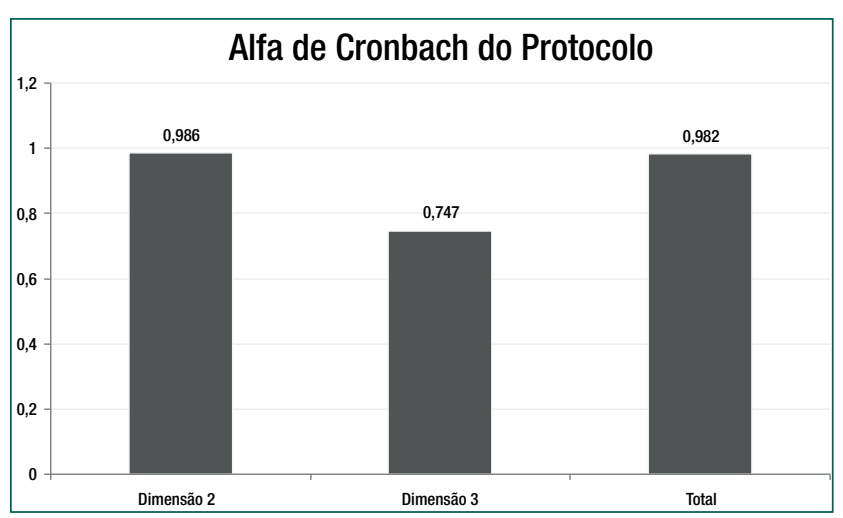

Figura 1. Percentual de Confiabilidade do IAE-ENF (Alfa de Cronbach) e suas respectivas Dimensões

A dimensão 2 do IAE-ENF buscou identificar aspectos relacionados à formação profissional do enfermeiro. Os itens que compuseram essa parte do instrumento foram construídos segundo as DCN/ ENF, incluindo os termos e conteúdo. Esta dimensão conteve 8 grupos de perguntas subdividas em subitens, em forma de escala Likert. Para a avaliação da média dos itens, foi considerada a pontuação máxima que foi de 5 pontos numa escala de 5/4/3/2/1 e a média total foi de 2.56 na IES-1, 2.13 na IES-2 e 2.27 na IES-3. A dimensão 3 do IAE-ENF foi composta por questóes semiestruturadas relacionadas à percepção do egresso quanto à formação profissional e à atuação no mercado de trabalho. Para as 7 (sete) questóes fechadas foi utilizada a escala de diferencial semântico, com apresentação numérica de 1 a 5 sendo 1 insuficiente e 5 suficiente. $O$ total desta dimensão foi calculado pela média das questốes que compóe esta dimensão e os resultados encontrados foram: 3.46 (IES-1), 3.27 (IES-2) e 3.35 (IES-3) respectivamente (Figura 2).

$\mathrm{Na}$ análise da validade de construto, por meio da análise fatorial exploratória, utilizou-se a medida de adequação de Kaiser-Meyer-Olkin (KMO) e Bartlett, a fim de verificar se a amostra do estudo estava adequada conforme mostra a tabela 1 . O resultado do 


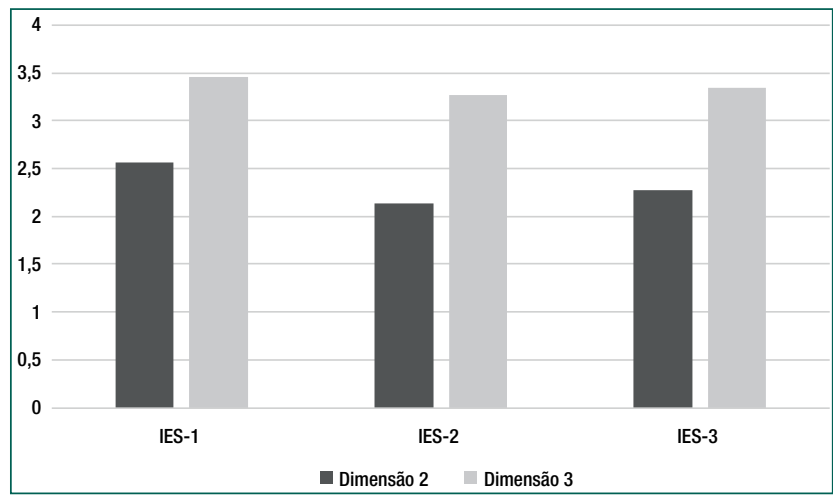

Figura 2. Distribuição das Médias das Dimensões 2 e 3 do IAE -ENF

Tabela 1. Teste de KMO e Bartlett para Análise Fatorial

\begin{tabular}{lc}
\hline Teste & Resultado \\
\hline KM0 & 0,971 \\
Bartlett & $<0,001$ \\
\hline
\end{tabular}

KMO foi de 0,971 , demostrando que há correlação excelente entre as variáveis. $\mathrm{O}$ teste de esfericidade de Bartlett apresentou resultados significativos $(<0,001)$ para todos os construtos e as matrizes de correlação entre os itens de cada construto (Tabela 1).

$\mathrm{Na}$ tabela 2 apresenta-se os resultados da análise fatorial exploratória, considerando o número de fatores identificados no teste scree plot.

Tabela 2. Valores Próprios e Variabilidade dos Fatores do IAE-ENF

\begin{tabular}{lccc}
\hline Fatores & Eigenvalues & \% Variância & \% Acumulado \\
\hline Fator 1 & 31,72 & 52,0 & 52,0 \\
Fator 2 & 4,38 & 7,2 & 59,2 \\
Fator 3 & 3,13 & 5,1 & 64,3 \\
Fator 4 & 2,11 & 3,5 & 67,8 \\
Fator 5 & 1,57 & 2,6 & 70,3 \\
Fator 6 & 1,46 & 2,4 & 72,7 \\
Fator 7 & 1,19 & 2,0 & 74,7 \\
Fator 8 & 1,08 & 1,8 & 76,5 \\
\hline
\end{tabular}

Constata-se que o primeiro fator, que deteve $52 \%$ da variabilidade dos dados, foi composto por 22 itens. Entre estes, destaca-se o item "Assumir o compromisso ético, humanístico e social com o trabalho multiprofissional em saúde" com carga fatorial de 0,819. A análise de tais resultados permitiu observar que os fatores 1,2,3,4 e 5 foram compostos exclusivamente por itens da dimensão 2 (dois) do IAE-ENF, ou seja, referentes às questôes relativas à formação profissional do Enfermeiro nas DCN/ENF. O fator 6 (seis), composto apenas pelos itens da dimensão 3 (três) do IAE-ENF, totalizando 6 (seis) itens, fez referência à percepção do egresso quanto à formação acadêmica recebida pela IES e às necessidades do mercado de trabalho. $\mathrm{O}$ fator 7 (sete) correspondeu a três questóes da dimensão 2 (dois) do IAE-ENF relacionada a formação profissional e o fator 8 (oito) com uma questão da dimensão 3 (três) do IAE-ENF, relacionada a remuneração da categoria profissional. A versão ajustada após a análise fatorial encontra-se, na íntegra, no apêndice 1 .

\section{Discussão}

Este estudo emerge da necessidade de disponibilizar para a comunidade científica o primeiro instrumento validado que aborda, na íntegra, as habilidades e competências exigidas pelas DCN/ENF e a percepção do egresso quanto à formação profissional e à atuação no mercado de trabalho. Os resultados indicam que IAEENF, na fase atual de validação, apresenta resultados satisfatórios nos testes aos quais foi submetido na presente investigação, assim como no estudo anterior, no qual foi submetido à validação de conteúdo. ${ }^{(12)}$

O IAE-ENF é um instrumento que segue critérios teóricos e metodológicos, garantindo um modelo fatorial adequado. A análise fatorial tem esse objetivo, investigar os elementos subjacentes em uma determinada matriz de dados por meio de técnicas multivariadas. Essas etapas são importantes para comprovar a estrutura interna e revelar a precisão dos construtos do instrumento. ${ }^{(20)}$

Inicialmente, os itens do IAE-ENF foram submetidos a análise da Medida Kaiser-Meyer-Olkin (KMO), para confirmação das correlaçóes entre os itens, apresentando bom ajuste para o modelo com oito domínios com $(0,97)$. Ainda, o Teste de Esfericidade de Bartlett, que verifica a significância das correlaçóes em uma matriz de dados, no qual o IAE-ENF demostrou correlaçóes entre os itens com $(<0,001)$, viabilizando assim, a realização da análise fatorial, assim como em outros estudos de validação de instrumentos que apresentaram valores semelhantes a este estudo. ${ }^{(21,22)}$

Confirmado a adequabilidade da análise fatorial para os itens do IAE-ENF, investigou-se a presença de mais de um fator na matriz geral de dados, me- 
diante a Análise dos Componentes Principais pelo teste do scree plot, com o objetivo de reduzir o número de itens, levando em consideração a variância das variáveis nos diferentes grupos estudados. Os resultados encontrados foi de uma matriz com oito fatores que explicaram $76,5 \%$, o que considera-se um valor bom, sendo que cada um dos demais fatores mostrou autovalores maiores do que 1 . $^{(18,20)}$

Quanto aos aspectos analisados com relação a formação e atuação do Enfermeiro, que corresponde a uma análise do conceito abstrato do que é esperado para a formaçáo profissional, a validação de construto do IAE-ENF investigou as relaçóes e a necessidade de adequação do IAE-ENF para medir esses aspectos, no entanto, decidiu-se por permanecer todos os itens já que os resultados são satisfatórios para essa matriz.

As medidas estatísticas para validar o construto do IAE-ENF em cada uma das três dimensóes originalmente propostas (ou seja, características sociodemográficas, formação profissional e atuação profissional) evidenciam bom entrosamento entre os itens, embora, as dimensões 2 e 3 provarem maior afinidades, confirmando a coerência dos itens propostos no instrumento original. ${ }^{(12)}$

Em relação à confiabilidade do IAE-ENF, os valores encontrados para o coeficiente Alpha de Cronbach foi elevado, indicando boa consistência interna. Em relação à estabilidade, os coeficientes de correlação intraclasse total de 94\% evidenciou forte concordância entre as mediçôes, e, quando analisadas separadamente, as dimensóes dois e três apresentaram valores superiores a $0,70 .^{(17)}$

Considerando-se o acréscimo dos itens 20 e 25, para melhor direcionar as respostas, os quais não contribuíram para a elevação e redução da confiabilidade do instrumento, o Alfa de Cronbach não teve variação, permanecendo com o valor de 0,98 , caracterizando o instrumento como confiável, em sua versão final.

O que pode ser revisto na dimensão 1(um) do IAEENF, que aborda as características sociodemográficas, é o item referente a identidade biológica. No IAE-ENF consta "sexo" e foi observado que apenas 67 egressos $(17,67 \%)$ responderam esse item. Nessa perspectiva, considera-se oportuna a reflexão sobre essa variável na construção de futuros projetos de pesquisa, acrescentando-se mais opçóes, incluindo a possibilidade livre de autodeclaração, pois o direito à expressão de gênero assumiu grande relevância na contemporaneidade. ${ }^{(23)}$

Posto isto, o instrumento denominado IAEENF ficou constituído por oito domínios, divididos em três dimensões assim descritas: dimensão $1 \mathrm{com}$ 23 itens relacionados as características sociodemográficas; dimensão 2, com oito grupos de questóes referentes aos requisitos contidos nas DCN/ENF e por fim, dimensão 3 com oito questóes referentes a atuação profissional e inserção do mercado de trabalho, sendo, duas destas dissertativas (Apêndice 1).

Os valores de Alpha de Cronbach encontrados neste estudo ficou acima do valor obtido no instrumento original. ${ }^{(12)}$ Quando analisados separadamente por dimensão, dois e três, observa-se que os valores de Alpha de Cronbach evidenciaram boa confiabilidade dos dados. Esses dados, confirmam que o IAE-ENF produz resultados consistentes e coerentes a partir das pontuaçóes propostas. Os achados, corroboram com outros estudos, nos quais afirma-se que um valor de, pelo menos 0,70 reflete uma confiabilidade aceitável. ${ }^{(24-26)}$

Em relação aos oito domínios do IAE-ENF, observou-se que a maioria corresponde a dimensão dois do instrumento em que todos os itens estão relacionados aos requisitos contidos na DCN/ENF. Em seguida, a dimensão três, em que os itens estão relacionados a atuação do Enfermeiro e a inserção no mercado de trabalho.

Considerando a utilização de técnicas semelhantes às adotadas neste estudo, também sugeridas em outros estudos de validação estas definem que além dos processos de avaliação para os quais o instrumento é submetido, a adequação de uma nova versão do instrumento merece reflexóes quanto a avaliação conceitual dos itens pela população-alvo e o diálogo com o autor do instrumento original quanto a pertinência dos ajustes e modificaçóes propostas. ${ }^{(15,20)} \mathrm{Na}$ presente investigação, houve consenso entre os autores para as alteraçóes realizadas.

A limitaçáo do presente estudo relaciona-se a não inclusão de participantes representativos de todos os estados da federaçáo brasileira, o que gera a necessidade de realização de teste de confiabilidade para sua aplicação em outros grupos populacionais. Quanto a versão validada do IAE-ENF, sugere-se que, para as futuras aplicaçôes, substitua-se o item sexo, Dimensão 1, por gênero 
e que se acrescente mais um item ( $3^{\mathrm{a}}$ opção), com um espaço livre para autodeclaraçáo do respondente.

\section{Conclusão}

Os resultados lapidaram o instrumento original e evidenciaram que o IAE-ENF é confiável e válido para a avaliação do egresso de curso de graduação em Enfermagem, baseado nas Diretrizes Curriculares dos Cursos de Enfermagem, ano base 2001. As análises psicométricas mostraram resultados favoráveis com o Alfa de Cronbach, evidenciando forte consistência interna do instrumento. Em relação à estabilidade, os coeficientes de correlação intraclasse total evidenciaram forte concordância entre as mediçóes. Os resultados do KMO e o teste de Bartlett foram significativos para todos os construtos e as matrizes de correlação entre os itens de cada construto, evidenciando boa correlação entre os dados. Implicações para a prática: O IAE-ENF é um avanço do processo avaliativo de egressos bacharéis de Enfermagem, podendo ser utilizado em território nacional para análise individual, institucional ou para análises comparativas, constituindo oportunidade de obtençáo de dados a partir de uma matriz comum, baseada nas DCN/Enf, com múltiplas possibilidades de análises.

\section{Colaborações}

Aguiar KLA, Vieira MA e De Domenico EBL contribuíram com a concepção do estudo, análise e interpretação dos dados, redação do artigo, revisão crítica relevante do conteúdo intelectual e aprovação da versão final a ser publicada.

\section{Referências}

1. Florez-Nisperuza EP, Hoyos-Merlano AM. Una mirada cualitativa sobre la autoevaluación institucional desde la praxis universitaria. Rev Cient. 2020;37(1):78-89.

2. Brasil. Senado Federal. Secretaria Especial de Editoração e Publicações. Subsecretaria de Edições Técnicas. Lei de Diretrizes e Bases da Educação Nacional. Lei n 9.394, de 20 de dezembro de 1996. Estabelece as diretrizes e bases da educação nacional. Brasília (DF): Senado Federal; 2005 [citado 2020 Nov 23]. Disponível em: https://www2.senado.leg.br/bdsf/bitstream/handle/id/70320/65.pdf

3. Ré CR. Morinian complexity and the nursing curriculum. Invest Educ Enferm. 2020;38(2):e11

4. Lima LA, Andriola WB. Acompanhamento de egressos: subsídios para a avaliação de Instituições de Ensino Superior (IES). Avaliação. 2018;23(1):104-25.

5. Souza NV, Pires AS, Gonçalves FG, Tavares KF, Baptista AT, Bastos TM. Formação em enfermagem e mundo do trabalho: percepções de egressos de enfermagem. Aquichan. 2017;17(2):204-16.

6. Higa EF, Gomes R, Carvalho MH, Guimarães AP, Taipeiro EF, Hafner ML, et al. Perceptions of nursing alumni regarding the course contribution in providing health care. Texto Context Enferm. 2013;22(1):97-105.

7. Miranda CS, Pazello ET, Lima CB. Egressos como instrumento de avaliação institucional: uma análise da formação e empregabilidade dos egressos da fea-rp/usp. Rev GUAL. 2015;8(1):298-321.

8. Souza SN, Miyadahira AM. 0 desenvolvimento de competências no curso de graduação em enfermagem: percepção de egressos. Cienc Cuid Saude. 2012;11(Suppl):243-50.

9. Vieira MA, Souza e Souza LP, Ohara CV, De Domenico EB. Avaliação com egressos da graduação em enfermagem: publicações nacionais entre 2001-2011. Hist Enferm Rev Eletron. 2014;5(1):35-53.

10. Vieira MA, Lima CA, Martins AC, Domenico EB. National curriculum guidelines for the nursing graduation course: implications and challenges. Rev Pesq Cuid Fundam Online. 2020;12:1099-104.

11. Meira MD, Kurcgant P. Nursing education: training evaluation by graduates, employers and teachers. Rev Bras Enferm. 2016;69(1):10-5.

12. Vieira MA, Ohara CV, Domenico EB. The construction and validation of an instrument for the assessment of graduates of undergraduate nursing courses. Rev Lat Am Enfermagem. 2016;24:e2710.

13. Sullivan KM, Dean A, Soe MM. OpenEpi: a web-based epidemiologic and statistical calculator for public health. Public Health Rep. 2009;124(3):471-4.

14. Streiner DL, Norman GR. Health measurement scales: a practical guide to their development and use. 4th ed. New York: Oxford University Press; 2008. $431 \mathrm{p}$.

15. Valerio MA, Rodriguez N, Winkler $P$, Lopez J, Dennison M, Liang $Y$, et al. Comparing two sampling methods to engage hard-to-reach communities in research priority setting. BMC Med Res Methodol. 2016;16(1):146.

16. Souza AC, Alexandre NM, Guirardello EB. Psychometric properties in instruments evaluation of reliability and validity. Epidemiol Serv Saude. 2017;26(3):649-59.

17. Vaske JJ, Beaman J, Sponarski CC. Rethinking internal consistency in Cronbach's Alpha. Leis Sci. 2017;39(2):163-73.

18. Watkins MW. Exploratory factor analysis: a guide to best practice. J Black Psychol. 2018;44(3):219-46.

19. Ferraz AS. Psicometria. Aval Psicol. 2016;15(1):129-31.

20. Grieder S, Grob A. Exploratory factor analyses of the Intelligence and Development Scales-2: implications for theory and practice. Assessment. 2020;27(8):1853-69.

21. Santos MC, Wechsler SM. Escala de otimismo para adultos: Construção e validação. Psico-USF. 2020;25(1):89-100

22. Araújo PR, Lima FE, Ferreira MK, Oliveira SK, Carvalho RE, Almeida PC. Medication administration safety assessment tool: construction and validation. Rev Bras Enferm. 2019;72(2):329-36. 
23. Alves RC, Silva EL. Universidade, gênero e sexualidade: experiências curriculares e formativas de estudantes não heterossexuais na UFRB. Gênero. 2016;17(1):83-98

24. Jain S, Angural V. Use of Cronbach's Alpha in dental research. Med Res Chron. 2017;4(3):285-91.
25. Moreira CB, Fernandes AF, Champion V, Dahinten VS, Vila VS, Howard AF, et al. Champion's Health Belief Model Scale Validity Evidence for Brazil. Acta Paul Enferm. 2020;eAPE20180264.

26. Taber KS. The use of Cronbach's Alpha When Developing and Reporting Research Instruments in science education. Res Sci Educ. 2018;48(6):1273-96.

\section{Apêndice 1. Instrumento IAE-ENF}

\section{DIMENSÃ0 1: CARACTERISTICAS SÓCIODEMOGRÁFICAS E INSERÇÃO NO MERCADO DE TRABALHO}

Etnia: [ Amarelo [ Branco [ Indígena [ Negro [ Pardo

Sexo: [ Masculino [ Feminino

Ano de Nascimento: _____

Ano/Semestre de conclusão do Curso de Graduação em Enfermagem: _ _ _

Assinale a natureza jurídica da Instituição de Ensino Superior onde concluiu sua Graduação em Enfermagem: — Pública [ Privada/Particular [ Outro. Qual?

Assinale o turno no qual realizou sua Graduação em Enfermagem: [ Integral [ Diurno [ Noturno

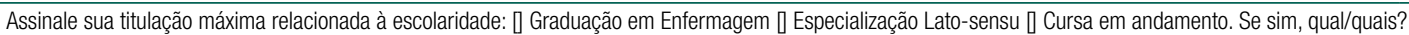

ano de conclusão:

Mestrado Acadêmico: [ Sim [ Em andamento / Se sim, citar Ano de conclusão:

Mestrado Profissional: — Sim \ Em andamento / Se sim, citar Ano de conclusão:

Doutorado: — Sim [ Em andamento / Se sim, citar Ano de conclusão: _. _ _ _

Pós-doutorado: [ Sim [ Em andamento / Se sim, citar Ano de conclusão:

Possui formação anterior na área da Enfermagem? Se sim, assinalar: \ Auxiliar de Enfermagem — Técnico de Enfermagem.

Você, como enfermeiro (a), possui emprego na área da Enfermagem? — Sim, em um local de trabalho. [ Sim, em mais de um local de trabalho. Quantos?

estou desempregado (a). [ Não, pois trabalho em outra área/profissão. Especifique qual:

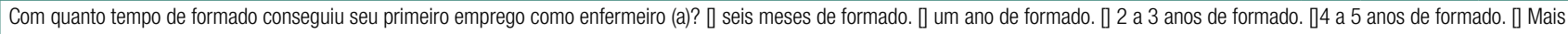
de seis anos de formado

Assinale como conseguiu ingressar no emprego atual como enfermeiro (a). . Concurso público. [ Processo seletivo. [ ] Contato direto com a instituição. [ Indicação de profissionais da

área. [ Indicação de amigos/parentes. [ Outros. Especifique:

Como enfermeiro (a), qual a carga horária total semanal de trabahho no seu emprego? [ ] A Aé 20 horas. [ De 21 a 36 horas. [ De 37 a 44 horas. [ De 45 a 60 horas. [ Acima de 61 horas.

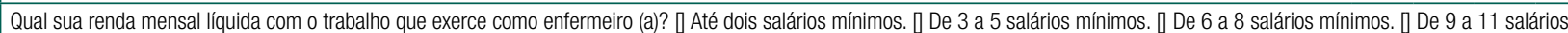
mínimos. \A Acima de 12 salários mínimos.

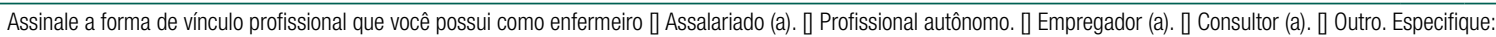

Em relação ao seu emprego atual como enfermeiro (a), assinale o cargo que exerce. [ Gerencial. [ ]Assistencial. [Educacional. [ Pesquisador (a). [ Consultor (a) ou Assessor (a). [0utra. Especifique

Em que modalidade de atenção à saúde você exerce seu emprego como enfermeiro (a)? — Ambulatório de Especialidades. —Área de Urgência/ Emergência. \ Auditoria em Saúde/Enfermagem.

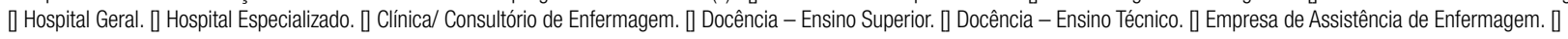

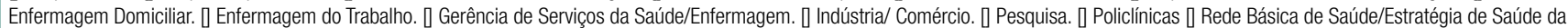
Família. [ Unidade de Pronto Atendimento. [ Unidade de Saúde tradicional. Outras. Especifique:

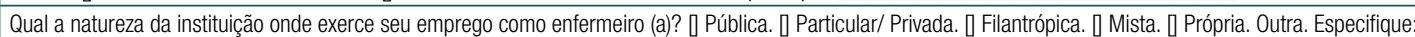

Você realizou ou está realizando outro curso? [ Sim \ Não Se sim, citar Qual?

\section{DIMENSÃO 2: AVALIAÇÃO DO PROCESSO DE FORMAÇÃO PROFISSIONAL}

Caríssimo (a) Egresso (a),

Os Cursos de Enfermagem respeitam as Diretrizes Curriculares Nacionais e devem formar Enfermeiros "com formação generalista, humanista, crítica e reflexiva. Profissional qualificado para o exercício da Enfermagem, com base no rigor científico e intelectual e pautado em princípios éticos. Capaz de conhecer e intervir sobre os problemas/situações de saúde-doença mais prevalentes no perfil epidemiológico nacional, com ênfase na sua região de atuação, identificando as dimensões biopsicossociais dos seus determinantes. Capacitado a atuar, com senso de responsabilidade social e compromisso com a cidadania, como promotor de saúde integral do ser humano" (BRASIL, 2001 p.1).

Considerando esta prerrogativa, leia e responda o questionário a seguir, circulando ao redor do número da escala que melhor indicar a sua percepção em relação ao item perguntado. Faça apenas um círculo por item perguntado.

Use os CÓDIGOS: CT- Concordo Totalmente. C- Concordo. IImparcial (Nem concordo, nem discordo). D- Discordo. DT - Discordo Totalmente.

1. 0 Curso de Graduação em Enfermagem preparou você para exercer atividades profissionais inerentes a sua área de atuação para ser capaz de: Competências e habilidades

1.1 No processo de saúde-doença, se responsabilizar pela qualidade da assistência/cuidado de enfermagem em seus diferentes níveis de atenção à saúde, com ações de promoção, prevenção, proteção e reabilitação da saúde, na perspectiva da integralidade da assistência.

1.2 Responder às especificidades regionais de saúde através de intervenções planejadas estrategicamente, nos níveis de promoção, prevenção e reabilitação da saúde.

1.3 Prestar cuidados de enfermagem compatíveis com as diferentes necessidades apresentadas pelo indivíduo.

1.4 Prestar cuidados de enfermagem compatíveis com as diferentes necessidades apresentadas pela família.

1.5 Prestar cuidados de enfermagem compatíveis com as diferentes necessidades apresentadas pela população.

1.6 Atuar nos programas de assistência integral à Saúde da Mulher.

1.7 Atuar nos programas de assistência integral ao Recém-Nascido.

1.8 Atuar nos programas de assistência integral à Saúde da Criança.

1.9 Atuar nos programas de assistência integral à Saúde do Adulto.

1.10 Atuar nos programas de assistência integral à Saúde do Idoso.

1.11 Utilizar referenciais teóricos e metodológicos, com ênfase no Processo de Enfermagem. 
Continuação.

2. 0 Curso de Graduação em Enfermagem instrumentalizou você para assistir o ser humano na sua integralidade, e assim, ser capaz de:

Competências e habilidades

2.1 Reconhecer a saúde como direito e condições dignas da vida.

2.2 Atuar de forma a garantir a integralidade da assistência, entendida como conjunto articulado e contínuo das ações e serviços preventivos e curativos,

individuais e coletivos, exigidos para cada caso, em todos os níveis de complexidade do sistema.

2.3 Atuar profissionalmente, compreendendo a natureza humana em seu ciclo de vida, de acordo com as características biopsicossociais e espirituais, e as

necessidades de cuidado.

2.4 Diagnosticar problemas de saúde e de enfermagem.

2.5 Tomar decisões e intervir sobre os problemas de saúde e de enfermagem diagnosticados.

2.6 Promover estilos de vida saudáveis, conciliando as necessidades tanto dos seus usuários quanto as de sua população adstrita, atuando como agente de transformação social.

2.7 Cuidar da própria saúde física e mental e buscar seu bem-estar, como cidadão e como enfermeiro(a).

3. 0 Curso de Graduação em Enfermagem fundamentou você, acerca das questões éticas ao exercício da profissão, para ser capaz de:

Competências e habilidades

3.1 Respeitar os princípios éticos, legais e humanísticos da profissão.

3.2 Reconhecer-se como coordenador do trabalho da equipe de enfermagem e responsável pelas ações determinadas aos membros da equipe de enfermagem.

3.3 Assumir o compromisso ético, humanístico e social com o trabalho multiprofissional em saúde.

3.4 Gerenciar o processo de trabalho em enfermagem com princípios de Ética e de Bioética.

3.5 Respeitar o direito do usuário à escolha e autodeterminação no cuidado de enfermagem e de sua saúde.

3.6 Respeitar a privacidade do usuário assegurando sigilo e segurança da informação adquirida em situação profissional.

4. 0 Curso de Graduação em Enfermagem preparou você tecnicamente e cientificamente, para ser capaz de:

Competências e habilidades

4.1 Coletivizar conhecimento técnico-cientifico que confira qualidade ao exercício profissional.

4.2 Planejar e/ou participar de pesquisas e/ou outras formas de produção do conhecimento que objetivem a qualificação da prática profissional.

4.3 Aplicar princípios científicos nas atividades profissionais.

4.4 Aplicar os princípios da Prática Baseada em Evidências no exercício profissional.

\section{0 Curso de Graduação em Enfermagem incentivou o exercício da cidadania para você ser capaz de:}

Competências e habilidades

5.1 Compreender a política de saúde no contexto das politicas sociais, reconhecendo os problemas e necessidades de saúde da população.

5.2 Reconhecer o papel social do enfermeiro para atuar em atividades de política e planejamento em saúde.

5.3 Interferir na dinâmica de trabalho institucional, reconhecendo-se como agente desse processo, com direitos e deveres.

5.4 Coordenar o processo de cuidar em enfermagem, considerando as atividades próprias da profissão.

6. 0 Curso de Graduação em Enfermagem preparou você para desenvolver atividades de ensino e ser capaz de:

Competências e habilidades

6.1 Atuar como sujeito no processo de formação de recursos humanos nos níveis superior e técnico.

6.2 Planejar, implementar e participar de programas de Educação Permanente dos trabalhadores de enfermagem e de saúde.

6.3 Planejar, implementar e avaliar programas de educação e promoção à saúde, considerando a especificidade dos usuários, famílias e população.

7. 0 Curso de Graduação em Enfermagem preparou você para trabalhar em equipe e ser capaz de:

Competências e habilidades

7.1 Coordenar as atividades da equipe de enfermagem.

7.2 Administrar conflitos entre os membros da equipe de enfermagem e multiprofissional.

7.3 Planejar e liderar ações que promovam a qualidade do trabalho em equipe.

8. 0 Curso de Graduação em Enfermagem forneceu subsídios para você realizar atividades de gestão em saúde e ser capaz de:

Competências e habilidades

8.1 Participar da cogestão do processo de trabalho em saúde.

8.2 Discutir os problemas e planos de intervenção para buscar soluções coletivas.

8.3 Estabelecer cooperação com a equipe de enfermagem e multiprofissional.

8.4 Responsabilizar-se pela qualidade dos cuidados prestados aos usuários, famílias e população.

8.5 Conduzir as análises de custo-efetividade, custo-benefício e custo utilidade de produtos e procedimentos em saúde.

8.6 Compatibilizar as características profissionais dos agentes da equipe de enfermagem às diferentes demandas dos usuários.

8.7 Reconhecer as relações de trabalho e sua influência na saúde.

\section{DIMENSÃO 3 - AVALIAÇÃO DA ATUAÇÃO PROFISSIONAL}

Caríssimo (a) Egresso (a),

Para você responder a DIMENSÃO 3 irá utilizar uma Escala do tipo Diferencial Semântico. Suas respostas devem ser registradas em uma escala de 1 a 5, sendo que a sua escolha é mais negativo quanto mais próximo do número 1 você assinalar e é mais positiva se você assinalar mais próximo do número 5.0 número 3 representa à média.

1. Descreva como você tem vivenciado a relação entre a sua formação acadêmica, recebida no Curso de Graduação em Enfermagem, e as exigências do mercado de trabalho no seu principal emprego.

2. Como você avalia as experiências práticas (estágios) que realizou na Graduação em Enfermagem em relação ao preparo para o exercício profissional?

3. Qual sua percepção em relação ao processo de Avaliação adotado na Graduação em Enfermagem que realizou?

4. Como enfermeiro (a), qual sua avaliação acerca de sua remuneração na Enfermagem?

5. 0 quanto você está satisfeito com as atividades profissionais que exerce na área da Enfermagem?

6. 0 quanto você está satisfeito em ser enfermeiro (a)?

7. Descreva as Potencialidades do Curso?

8. Descreva as Fragilidades do Curso?

\begin{tabular}{|l|l|l|l|l|}
\hline 1 & 2 & 3 & 4 & 5 \\
\hline 1 & 2 & 3 & 4 & 5 \\
\hline 1 & 2 & 3 & 4 & 5 \\
\hline 1 & 2 & 3 & 4 & 5 \\
1 & 2 & 3 & 4 & 5 \\
\hline 1 & 2 & 3 & 4 & 5 \\
\hline \multicolumn{6}{|c|}{ Questão aberta } \\
\hline \multicolumn{6}{|c|}{ Questão aberta }
\end{tabular}

